\title{
Apoptotic cell-derived ICAM-3 promotes both macrophage chemoattraction to and tethering of apoptotic cells
}

\author{
EE Torr ${ }^{1}$, DH Gardner ${ }^{1}$, L Thomas ${ }^{1}$, DM Goodall ${ }^{2}$, A Bielemeier ${ }^{1}$, R Willetts ${ }^{1}$, HR Griffiths ${ }^{1,3}$, LJ Marshall ${ }^{1,3}$ and A Devitt ${ }^{\star 1,3}$
}

A wide range of molecules acting as apoptotic cell-associated ligands, phagocyte-associated receptors or soluble bridging molecules have been implicated within the complex sequential processes that result in phagocytosis and degradation of apoptotic cells. Intercellular adhesion molecule 3 (ICAM-3, also known as CD50), a human leukocyte-restricted immunoglobulin super-family (IgSF) member, has previously been implicated in apoptotic cell clearance, although its precise role in the clearance process is ill defined. The main objective of this work is to further characterise the function of ICAM-3 in the removal of apoptotic cells. Using a range of novel anti-ICAM-3 monoclonal antibodies (mAbs), including one (MA4) that blocks apoptotic cell clearance by macrophages, alongside apoptotic human leukocytes that are normal or deficient for ICAM-3, we demonstrate that ICAM-3 promotes a domain 1-2-dependent tethering interaction with phagocytes. Furthermore, we demonstrate an apoptosis-associated reduction in ICAM-3 that results from release of ICAM-3 within microparticles that potently attract macrophages to apoptotic cells. Taken together, these data suggest that apoptotic cell-derived microparticles bearing ICAM-3 promote macrophage chemoattraction to sites of leukocyte cell death and that ICAM-3 mediates subsequent cell corpse tethering to macrophages. The defined function of ICAM-3 in these processes and profound defect in chemotaxis noted to ICAM-3-deficient microparticles suggest that ICAM-3 may be an important adhesion molecule involved in chemotaxis to apoptotic human leukocytes.

Cell Death and Differentiation (2012) 19, 671-679; doi:10.1038/cdd.2011.167; published online 25 November 2011

Cells undergoing programmed cell death (apoptosis) are efficiently removed by phagocytes in a process important for development, tissue maintenance and control of immune responses. Whereas neighbouring viable cells may remove low levels of local cell death, professional recruited macrophages deal with high levels of apoptotic death. Failure to effectively clear dying cells has been implicated in a range of pathological scenarios. ${ }^{1-3}$

The molecular mechanisms that underlie phagocyte recognition and clearance of apoptotic cells are still under investigation, although a wide range of phagocyte receptors and a more limited number of apoptotic cell-surface molecules have been defined. ${ }^{2,4}$ These molecules participate in a multistage process that, at least in the case of recruited professional phagocytes, involves recruitment through chemotaxis $^{5-8}$ before apoptotic cell recognition, tethering and phagocytosis, all of which are accompanied by immunomodulatory signalling. The most widely established change at the apoptotic cell surface is exposure of the aminophospholipid phosphatidylserine (PS) that mediates clearance. ${ }^{9-11}$ However, other less well-defined changes indicate an involvement of sugars, ${ }^{12-14}$, PS oxidation ${ }^{15}$ and exposure of intracellular components (e.g., annexin I). ${ }^{16}$ Previous work suggested that the immunoglobulin super-family (IgSF) member intercellularadhesion molecule (ICAM)-3, expressed on human leukocytes, becomes functionally altered during apoptosis to mediate clearance of apoptotic leukocytes through suggested generation of neo-epitopes (i.e., 'eat me' signals). ${ }^{17}$ However, more recently, a study of CD31, another IgSF member, suggests that molecules on viable cells may provide inhibitory signals $^{18}$ that are lost during apoptosis, permitting viable cellular interactions (e.g., CD31-CD31 in trans) to become functional phagocytic interactions. ${ }^{18-20}$ CD47 may also mediate such inhibitory signals. ${ }^{21-23}$ It seems likely that loss of inhibitory signals and gain of neo-epitopes occur at the apoptotic cell surface and together facilitate cell removal. However, the role and nature of apoptotic cell-associated ICAM-3 in the multistep process of apoptotic cell clearance have remained elusive.

Here we show apoptotic cell-associated ICAM-3 functions in tethering apoptotic cells to phagocytes. However, during apoptosis it is known that levels of surface proteins may be reduced through loss of membrane into apoptotic bodies (microparticles). ${ }^{24}$ Such microparticles from apoptotic B cells have been shown to have strong chemoattractive properties ${ }^{24}$ in a manner dependent upon the chemokine fractalkine

\footnotetext{
${ }^{1}$ School of Life and Health Sciences, Aston University, Birmingham, UK; ${ }^{2}$ Division of Immunity and Infection, University of Birmingham, Birmingham, UK and ${ }^{3}$ Aston Research Centre for Healthy Ageing, Aston University, Birmingham, UK

${ }^{*}$ Corresponding author: A Devitt, School of Life and Health Sciences, Aston Research Centre for Healthy Ageing, Aston University, Birmingham, West Midlands B4 7ET, UK. Tel: + 44121204 4165; Fax: + 44121204 4187; E-mail: a.devitt1@aston.ac.uk

Keywords: monocytes/macrophages; ICAM-3; apoptosis; inflammation; chemotaxis; phagocytosis

Abbreviations: AxV, annexin V; BL, Burkitt's lymphoma; Bcl-2, B-cell lymphoma 2; DAPI, 4,6-diamidino-2-phenylindole; EV, electronic volume; FCS, fetal calf serum; FITC, fluorescein isothiocyanate; FS, forward scatter; GFP, green fluorescent protein; HEK, human embryonic kidney; ICAM, intercellular adhesion molecule; IgSF, immunoglobulin super-family; mAb, monoclonal antibody; MFI, mean fluorescence intensity; PBS, phosphate-buffered saline; PE, R-phycoerythrin; PI, propidium iodide; PMA, phorbol-12-myristate-13-acetate; PS, phosphatidylserine; SS, side scatter; WT, wild-type

Received 08.2.11; revised 30.8.11; accepted 03.10.11; Edited by P Vandenabeele; published online 25.11.11
} 
(CX3CL1), which acts as a 'find me' signal, and its cognate receptor (CX3CR1). ${ }^{25}$ Here we show that rapid and substantial loss of ICAM-3 from the surface of apoptotic leukocytes occurs via microparticle shedding and these microparticles are strongly chemoattractive in a manner dependent upon ICAM-3. Together with the established role of ICAM-3 as an important adhesion molecule involved in immune synapse formation, our data provide new insight into the mechanisms of chemoattraction of phagocytes to apoptotic cells and identify ICAM- 3 as a key adhesion molecule within the process.

\section{Results}

Interaction of apoptotic cells with phagocytes is inhibited by ICAM-3 mAb MA4. Moffatt et al. ${ }^{17}$ characterised two, now unavailable, anti-ICAM-3 monoclonal antibodies (mAbs: BU68 and 3A9) capable of blocking interaction of apoptotic cells with phagocytes showing that they were reactive to the membrane-distal domains of ICAM-3 (domains 1 and 2). Here a panel of novel mAbs raised from mice immunised with ICAM-3-transfected human embryonic kidney (HEK) cells were screened by ELISA against recombinant ICAM-3 (domains 1 and 2)-Fc fusion protein and CD14-Fc as a control (Supplementary Figure 1). We identified 17 anti-ICAM-3 mAbs specific for domains 1 and 2.

Using an established model system of specific apoptotic cell clearance, ${ }^{26,27}$ we screened these anti-ICAM-3 mAbs and identified one, MA4, that robustly inhibited apoptotic cell interaction with phagocytes (Figures $1 \mathrm{a}$ and $\mathrm{b}$ ). The observed degree of inhibition with MA4 (59 $\pm 14 \%)$ was in line with that seen previously with ICAM-3 mAbs BU68 and 3A9. ${ }^{17}$ Other mAbs (e.g., BH10 and GD12) of the same isotype (murine $\lg \mathrm{G} 1 / \kappa)$ were also screened but failed to inhibit clearance. Further analyses indicated that MA4, BH10 and GD12 were all capable of reacting with full-length ICAM-3 (domains 15) that was denatured and reduced (assessed by western blotting) or native and cell-associated ICAM-3 (assessed by flow cytometry) (Supplementary Figure 1).

These results confirm MA4 as a novel anti-ICAM-3 mAb that reacts with a probable linear epitope in domains 1 and 2 of ICAM-3, and is capable of inhibiting apoptotic cell interaction with phagocytes.

\footnotetext{
Apoptotic cells deficient for ICAM-3 exhibit reduced clearance by phagocytes. Earlier studies showed that the role of ICAM-3 on apoptotic cells in promoting clearance can be revealed through the use of inhibitory mAbs (Figure 1a, and see Moffatt et al. ${ }^{17}$ ) and by overexpression of ICAM-3 to promote clearance. ${ }^{17}$ Here we demonstrate an alternative and complementary method to reveal a role for ICAM-3 in apoptotic cell clearance. ICAM-3-deficient Mutu Burkitt's lymphoma (BL) cells or Jurkat $T$ cells (human leukocyte cell lines that normally express ICAM-3) were isolated following sequential flow cytometric sorting for very low/ negative expression levels of surface ICAM-3. The cellular proteome analysed by $2 \mathrm{D}$ gel electrophoresis ${ }^{28}$ was not significantly different between ICAM-3-deficient cells and
}

a

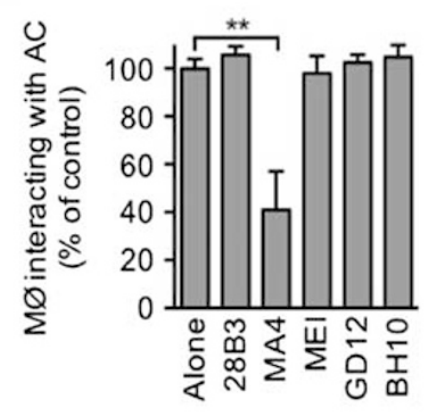

b

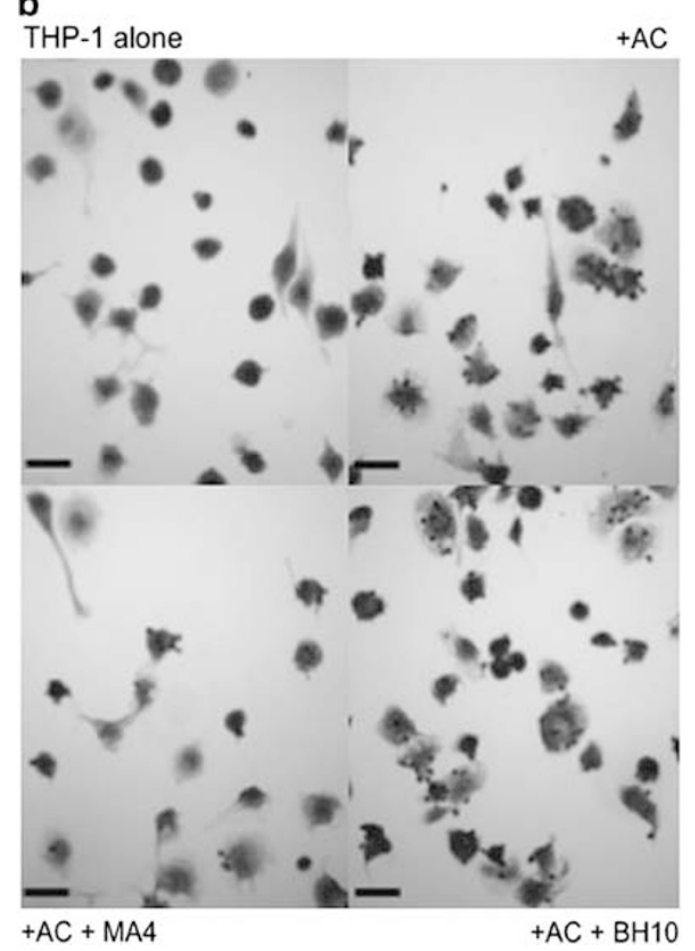

Figure 1 Inhibition of apoptotic cell interaction with THP-1 phagocytes by antiICAM-3 mAb MA4. (a) Mutu BL cells were induced to apoptosis by UV $\left(100 \mathrm{~mJ} / \mathrm{cm}^{2}\right)$ and co-cultured with THP-1 phagocytes $(\mathrm{M} \varnothing)$ in the presence of the indicated mAbs. The proportion of $M \varnothing$ interacting with (tethering and/or phagocytosing) apoptotic cells (ACs) was scored using light microscopy following Jenner/Giemsa staining. Data (mean \pm S.D.) from at least three separate experiments each carried out in quadruplicate are shown (data are normalised to the mAb-free co-culture ('Alone'). (b) Microscopic appearance of a THP-1 phagocyte culture or co-cultures of THP-1 and ACs in the presence or absence of the indicated mAb (MA4 or BH10). Scale bar $=50 \mu \mathrm{m} .{ }^{*} P<0.01$ ANOVA with Dunnett's post-hoc test

their ICAM-3-replete counterparts. Such ICAM-3-deficient cells exhibited reduced ability to interact with THP-1 phagocytes when compared with their ICAM-3-replete counterparts (Figure 2a). Similar results were noted with primary human monocyte-derived macrophages (Supplementary Figure 2). Detailed analysis of apoptotic cell death indicated that both populations of cells died with the same kinetics and to the same extent irrespective of their ICAM-3 expression (Supplementary Figure 3). Furthermore, when apoptotic cells were incubated with phagocytes in the presence of MA4, only the recognition of ICAM-3-expressing cells was inhibited 
a

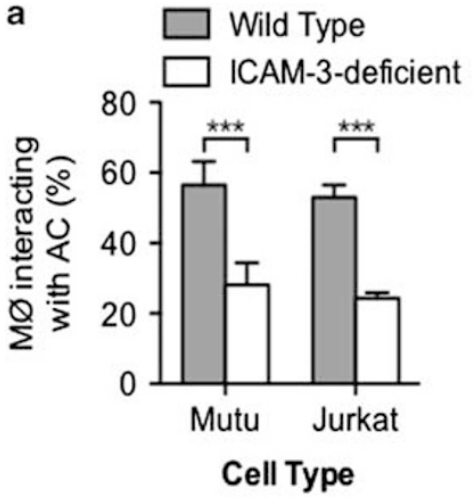

b

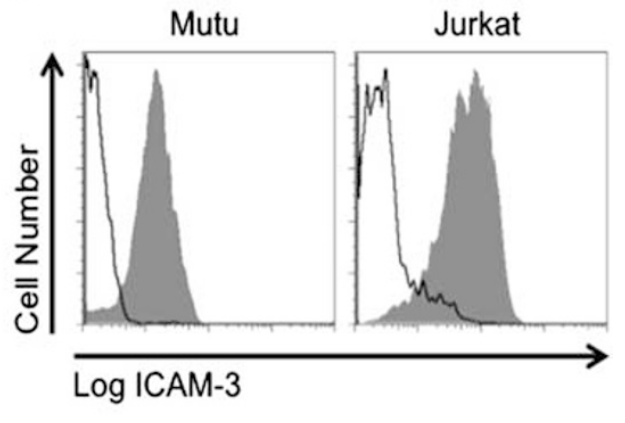

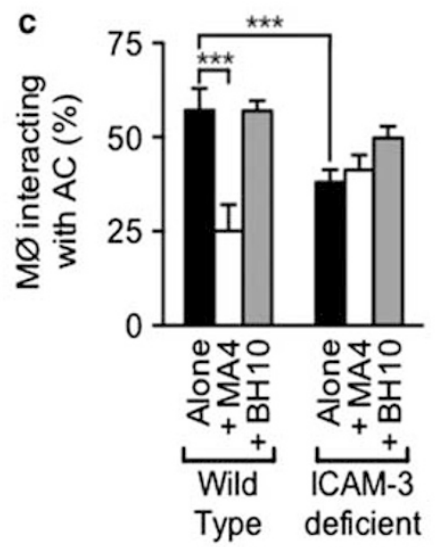

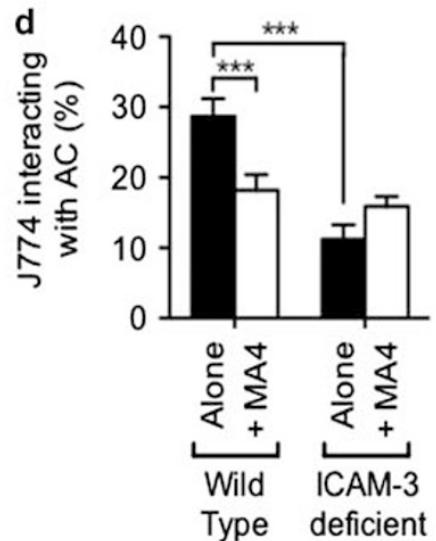

Figure 2 Interaction of apoptotic cells with human or mouse phagocytes is reduced in the absence of apoptotic ICAM-3. WT and ICAM-3-deficient BL and Jurkat cells were induced to apoptosis by UV (Mutu: $100 \mathrm{~mJ} / \mathrm{cm}^{2}$; Jurkat $150 \mathrm{~mJ} / \mathrm{cm}^{2}$ ) and co-cultured with THP-1 phagocytes (MØ) for $1 \mathrm{~h}$. The proportion of MØ interacting with (tethering and/or phagocytosing) apoptotic cells (ACs) was scored using light microscopy following Jenner/Giemsa staining (a). Expression of ICAM-3 on apoptotic target cells was assessed by indirect immunofluorescence. Flow cytometric histograms of anti-ICAM-3 mAb-stained WT (grey profiles) or ICAM-3-deficient (clear profiles) Mutu/BL or Jurkat cells are shown (b). The ability of the blocking anti-ICAM-3 mAb MA4 to modulate the capacity of apoptotic WT and ICAM-3-deficient BL cells to interact with THP-1 phagocytes (c) and $\mathbf{J 7 7 4}$ mouse phagocytes (d) was assessed. Data shown are mean \pm S.D. of replicate samples $(n=4)$ from one experiment (of at least four similar). ${ }^{* \star *} P<0.001$ ANOVA with Tukey's post-hoc test

(Figure 2c and d), supporting the specificity of effect by MA4. This phenomenon was evident irrespective of the method chosen for induction of apoptosis (UV (Figure 2), ionomycin or staurosporine (data not shown)).

It is notable that ICAM-3 mediates apoptotic human leukocyte clearance by murine macrophages (Figure $2 \mathrm{~d}$ ). These data demonstrate that the phagocyte receptor function for apoptotic ICAM-3 is present in mice where ICAM-3 is naturally absent. Taken together, these data further demonstrate a role for ICAM-3 in the interaction of apoptotic leukocytes with phagocytes, consistent with our previous reports, and that the receptor for apoptotic ICAM-3 likely recognises molecular changes that may not be restricted to ICAM-3. Indeed, these observations further support the notion that clearance mechanisms are highly conserved across species.

Apoptotic cell-associated ICAM-3 mediates tethering to phagocytes. The phagocytic removal of apoptotic cells is a complex multistep process. ${ }^{2}$ To address whether apoptotic cell-associated ICAM-3 functions as a ligand for tethering or engulfment, apoptotic leukocytes were co-cultured with phagocytes at $20^{\circ} \mathrm{C}$, permissive only for apoptotic cell tethering to phagocytes, ${ }^{29}$ and results were compared with assays at $37^{\circ} \mathrm{C}$, permissive for both tethering and phagocytosis. Here we show reduced tethering of ICAM-3-deficient BL cells with human phagocytes when compared with their ICAM-3-replete counterparts (Figure 3a). These data strongly implicate ICAM-3 as a tethering ligand for apoptotic cells, a function also demonstrated using mouse macrophages (Figure 3b).

ICAM-3 levels reduce during apoptosis due to shedding in microparticles. It is intriguing that ICAM-3 levels reduce during apoptosis given the emergence of a role for ICAM-3 in apoptotic cells. ${ }^{17}$ Mutu B cells provide a robust model for the analysis of early apoptosis by flow cytometry. Light scatter (forward scatter/electronic volume (FS/EV) versus side scatter (SS)) defines two cell populations (Figure 4a): region 1: smaller and more granular cells that are necrotic (including late apoptotic/secondary necrotic cells) and stain with the vital dye propidium iodide (PI); and region 2: larger and less granular cells (live or early apoptotic) where plasma membranes are intact and exclude PI. ${ }^{27,30}$ Region 2 cells (live/early apoptotic) are therefore viable or actively undergoing apoptosis (as detected by annexin $\mathrm{V}(\mathrm{AxV})$ staining and exclusion of $\mathrm{PI}$ ). This live/early apoptotic region 

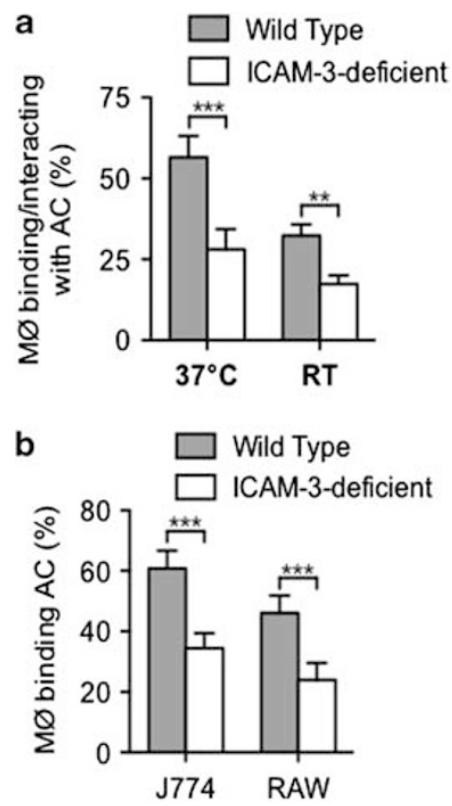

Figure 3 Tethering (binding) of apoptotic cells to human or mouse phagocytes is reduced in the absence of apoptotic cell ICAM-3. BL and Jurkat cells (WT or ICAM-3 deficient) were induced to apoptosis by UV (Mutu: $100 \mathrm{~mJ} / \mathrm{cm}^{2}$; Jurkat $150 \mathrm{~mJ} / \mathrm{cm}^{2}$ ). The capacity of THP-1 phagocytes to tether apoptotic cells (WT, grey bars; ICAM-3-deficient, open bars) was assessed at room temperature (nonpermissive for phagocytosis) and compared with interaction (binding and phagocytosis), which was assessed at $37^{\circ} \mathrm{C}$ (a). Similar tethering assays using $\mathrm{J774}$ and RAW mouse phagocytes are shown (b). Data shown are mean \pm S.D. of replicate samples $(n=4)$ from one experiment of at least three similar. ${ }^{\star} P<0.01$; ${ }^{* * *} P<0.001$ ANOVA with Tukey's post-hoc test

of a population of Mutu BL cells will be predominantly $\mathrm{AxV}+$ / $\mathrm{PI}-$ within $5-6 \mathrm{~h}$ of UV irradiation. With continued incubation, these cells change morphology to fall in region 1 (late apoptotic/necrotic) with apoptotic nuclear morphology. ${ }^{27,30}$

In line with previous work, ${ }^{17}$ we noted ICAM-3 reduced (by $30 \%$ ) in Mutu BL cells $24 \mathrm{~h}$ after apoptosis induction (mean fluorescence intensity (MFI) Mutu control $=50.3$ versus Mutu $24 \mathrm{~h}$ post $U V=35.2$. No such decrease was noted with apoptosis-resistant Mutu/B-cell lymphoma 2 (bcl-2) cells (MFI Mutu/bcl-2 control $=50$ versus Mutu/bcl-2 $24 \mathrm{~h}$ post $\mathrm{UV}=47.7)$. To exclude the effects of secondary necrosis or prolonged incubation in vitro, ICAM-3 levels on early apoptotic cells were further studied.

Here we demonstrate that ICAM-3 levels begin to reduce in Mutu cells (but not Mutu/Bcl-2 cells) while still confined to the live/early apoptotic region 2, suggesting that ICAM-3 levels reduce early in apoptosis (Figure $4 \mathrm{~b}$ ) and low ICAM-3 levels are most closely associated with PS exposure as detected with $A x V$-fluorescein isothiocyanate (FITC) (Figure 4c). All cells that show low expression of ICAM-3 are also exposing PS.

The mechanism of ICAM-3 reduction has not been reported. A number of possible explanations for this exist and are associated with monoclonal antibody detection of ICAM-3. Structural alterations to ICAM-3 (e.g., changes in glycosylation) may render mAbs unable to detect ICAM-3 even if present. Alternatively, molecular redistributions of ICAM-3 during apoptosis may result in epitope masking such that ICAM-3 is less detectable (via mAbs) rather than being reduced in levels. To address this possibility, we generated a green fluorescent protein (GFP)-tagged ICAM-3 (C terminal tag; intracellular) and expressed this in HeLa cells. These cells showed reduced levels of ICAM-3-GFP as apoptosis proceeded (Figure 4d), suggesting ICAM-3 loss. Together, the reduction in GFP and concomitant loss of cell volume (Figure 4d) suggest that ICAM-3 levels are unlikely to be reduced simply as a result of ICAM-3 cleavage from the cell, leaving behind the GFP tag and transmembrane domain. Loss of ICAM-3 as an explanation of the reduced ICAM-3 levels was also supported by the observation that lymphocyte ICAM-3 levels (detected using immunofluorescent flow cytometry) also showed ICAM-3 reduced with cell volume (Figure 4e). Apoptosis-induced reduction in cell volume is known to occur via a number of mechanisms including membrane loss through release of blebs to apoptotic bodies (membrane-enclosed apoptotic cell-derived material, particles also known as microparticles).

To test the hypothesis that ICAM-3 levels reduce during apoptosis as a result of shedding within microparticles, ICAM3-GFP-transfected HeLa cells were studied. Importantly, phagocytes cleared these cells in an ICAM-3-dependent manner (revealed by MA4 inhibition of the interaction; Supplementary Figure 4) indicating that GFP tagging of ICAM-3 did not disrupt the function of ICAM-3 in apoptotic cell clearance. Detailed microscopic analyses of HeLa-ICAM-3GFP cells throughout an established model of apoptosis ${ }^{31}$ revealed ICAM-3-GFP within blebs at the apoptotic cell surface (Figure 5a). These blebs appeared early (beginning 2 to $3 \mathrm{~h}$ post anisomycin) in the apoptosis programme and in some cases were large (up to $5 \mu \mathrm{m}$ diameter) and contained nuclear material (Figure 5a). Although HeLa cell apoptosis differs in respect of membrane blebbing to that seen in leukocytes, the blebbing noted within our studies equates to the second wave of HeLa cell blebbing that is similar to that seen in leukocytes. ${ }^{31}$ However, it is unclear if the observed blebs are released. In order to assess then if such relocation of ICAM- 3 occurs in our leukocyte model of apoptosis and to extend our observations to assess if these visualised blebs are released from dying cells, wild-type (WT) or ICAM-3deficient Mutu cells were induced to apoptosis and culture supernatants harvested. Using centrifugation to enrich for microparticles and western blotting to detect ICAM-3, our data indicate a clear release of ICAM-3 in microparticles (Figure 5b).

Microparticle release from dying $B$ cells has been reported and is accompanied by loss of cell-surface molecules, and those particles are reported to be chemoattractive for macrophages. ${ }^{24}$ More recently, microparticles from Mutu BL cells have been noted to have chemoattractive capacity to recruit macrophages to sites of cell death in a manner, at least partly, dependent upon the chemokine CX3CL1 and its receptor CX3CR $1 .{ }^{25}$

Given that cell recruitment often requires a chemokine and adhesion molecule to affect cell recruitment, and that we have here shown that ICAM-3 functions to tether dying cells to phagocytes, we reasoned that ICAM- 3 is a possible candidate adhesion molecule involved in microparticle interaction with macrophages, thus mediating chemoattraction. To assess 

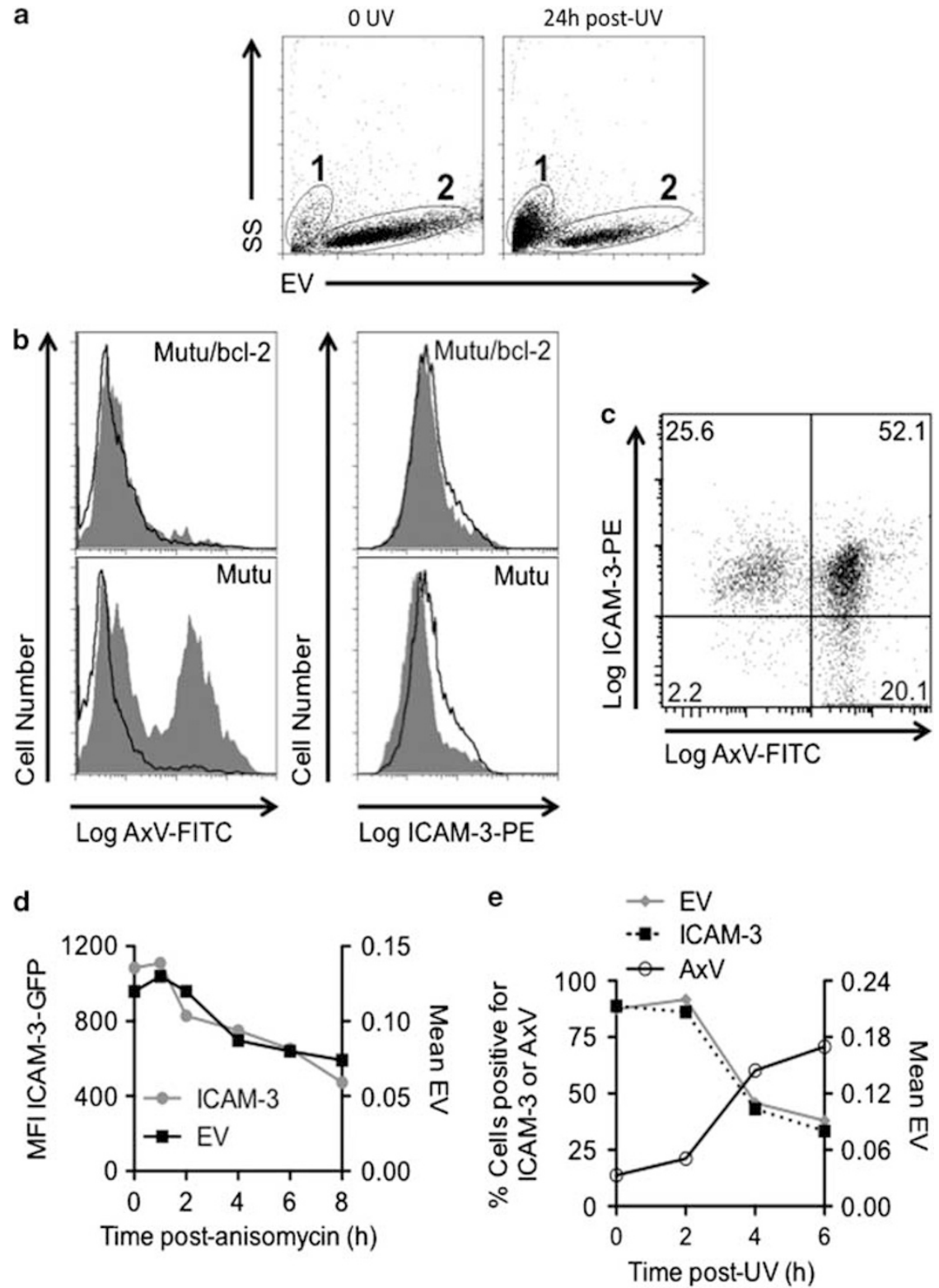

Figure 4 Cell-surface ICAM-3 levels reduce during apoptosis in line with a loss of cell volume. (a) Electronic volume versus side scatter flow cytometric dot plots of Mutu BL cells at $0 \mathrm{~h}$ (left) or $24 \mathrm{~h}$ (right) post UV. Region 1 cells are necrotic and late apoptotic whereas region 2 cells are live or early apoptotic. (b) Mutu BL cells or Bcl-2-transfected counterparts (Mutu/bcl-2) were irradiated with UV $\left(100 \mathrm{~mJ} / \mathrm{cm}^{2}\right)$ and $4 \mathrm{~h}$ post UV were assessed in parallel for PS exposure (using AxV-FITC staining) and ICAM-3 expression (using direct immunofluorescence with anti-ICAM-3-PE) of cells within zone 2 (live/early apoptotic cells). Grey profiles show staining of irradiated cells whereas clear profiles show nonirradiated cells. UV-treated Mutu but not Mutu/bcl2 cells show high level of PS exposure and reduced levels of ICAM-3. (c) UV-induced Mutu BL stained for both ICAM-3 expression (ICAM-3-PE) and PS exposure (AxV-FITC) shows PS-exposing cells (in region 2 - live/early apoptotic) preferentially express reduced ICAM-3. (d) HeLa cells expressing ICAM-3-GFP were assessed for cell volume and ICAM-3-GFP expression over time following induction to apoptosis with anisomycin $(5 \mu \mathrm{g} / \mathrm{ml})$. (e) Mutu BL cells were induced to apoptosis with UV and the percentage of cells positive for ICAM-3 (assessed using anti-ICAM-3-PE and flow cytometry) was compared with cell volume (electronic volume (EV)) and PS exposure (AxV). Data shown are collected from at least 5000 events and are representative of at least three similar experiments

this, microparticles from BL cells (WT or deficient for ICAM-3) were tested for their chemoattractive capacity in a microBoyden chamber. Our data revealed a potential for microparticles from BL cells WT for ICAM-3 to promote phagocyte movement (Figure 6a). Interestingly, microparticles released from ICAM-3-deficient BL cells were significantly less potent, suggesting a major role for ICAM-3 in this process. In order to address the possibility that microparticles from ICAM-3- replete cells were simply promoting chemokinesis rather than directional movement (chemotaxis), phagocytes were exposed to microparticles without a gradient (i.e., microparticles were present in both wells of the chamber). In this case, phagocyte movement was significantly reduced (Figure 6b), suggesting a gradient was necessary to elicit movement of phagocytes, and confirms the chemoattractive potential of microparticles from ICAM-3-replete cells. One 

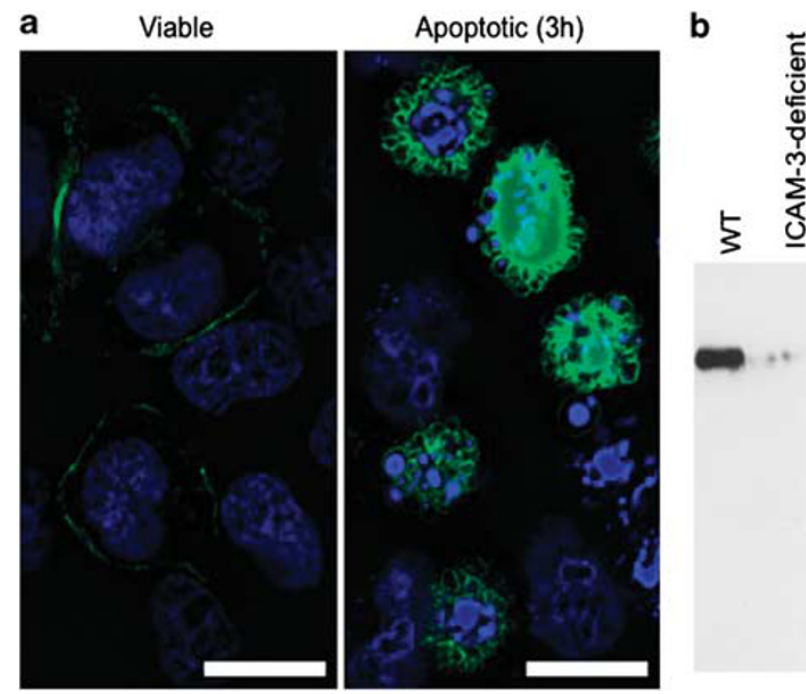

Figure 5 ICAM-3 is present in apoptotic blebs during zeiosis and is released from apoptotic cells in microparticles. (a) HeLa cells transfected with ICAM-3-GFP were imaged using epifluorescence microscopy following apoptosis induction with anisomycin $(5 \mu \mathrm{g} / \mathrm{ml})$. Cell nuclei were stained with DAPI $(250 \mathrm{ng} / \mathrm{ml})$ and optical sections photographed and deconvoluted with Volocity software (PerkinElmer). Images shown are noninduced and $3 \mathrm{~h}$ post anisomycin. Images were selected to demonstrate the location of ICAM-3. Apoptotic cells expressing high levels of ICAM-3-GFP are shown to clearly reveal the location of ICAM-3 on blebs. Quantitative data relating to ICAM-3 expression are provided in Figure 4. (b) Mutu BL cells (WT or ICAM-3 deficient) were UV induced to apoptosis and $16 \mathrm{~h}$ post-UV culture supernatants harvested. Cell bodies were removed and discarded following low-speed centrifugation $(7 \mathrm{~min} ; 350 \times \mathrm{g})$ and remaining microparticles purified through high-speed centrifugation. Pelleted microparticles were prepared and separated by PAGE and a western blot probed with anti-ICAM-3 mAb MA4. Scale bar $=16 \mu \mathrm{m}$

simple explanation of these results may be that cells WT for ICAM-3 died more quickly or to a greater extent, and thus were likely to generate more microparticles. Our studies indicated that the kinetics and extent of death within these two cell lines were equivalent. In order to assess generation of microparticles, supernatants from apoptotic cultures of WT or ICAM-3deficient cells, as used in the chemoattraction assays, were analysed by dynamic light scattering using a Zetasizer (Malvern Instruments, Malvern, UK). Such analyses measure particle diameter and our studies indicated no significant difference between the generated microparticles, with both cultures showing similar numbers and size distribution of the particles (WT: $186 \pm 26.2 \mathrm{~nm}$; ICAM-3 deficient: $206 \pm$ $47.7 \mathrm{~nm}$ ). Taken together, these data suggest that ICAM-3 in microparticles supports phagocyte chemoattraction.

\section{Discussion}

Herein we further characterise the role of ICAM-3, an established mediator of apoptotic cell clearance, ${ }^{17}$ in mediating this complex, multistage process that comprises, in vitro, recognition (to enable specific identification of dying cells), binding/tethering (a strong cell-cell interaction), phagocyte signalling (to initiate the next phase of the process), phagocytosis (reorganisation of cytoskeleton and membrane for uptake) and finally degradation. In vivo, however, it may be necessary for phagocytes to seek dying cells
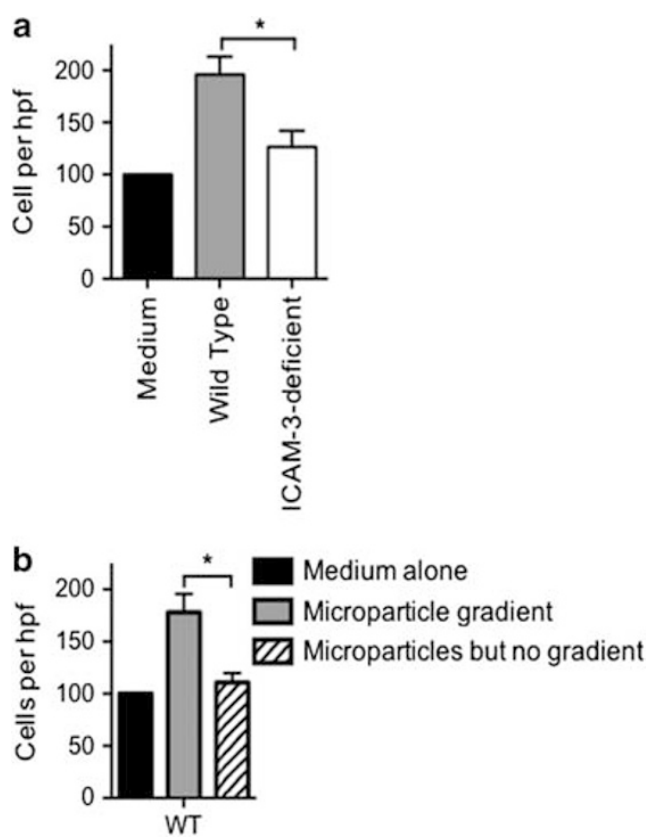

Figure 6 Microparticles from apoptotic cells mediate macrophage chemoattraction in an ICAM-3-dependent manner. Microparticles were prepared from cultures of apoptotic Mutu BL cells (WT or ICAM-3 deficient) at $16 \mathrm{~h}$ post UV. Supernatants were centrifuged to remove cell bodies $(7 \mathrm{~min} ; 350 \times \mathrm{g})$ and used neat. (a) Chemotaxis assay: the bottom chamber contained microparticles whereas the top chamber contained dihydroxyvitamin D3-stimulated THP-1 cells. Migration was allowed to proceed for $4 \mathrm{~h}$ before staining of migrated cells and quantitation by light microscopy. For each experimental treatment, four replicate wells were used and within each five high-power fields (HPFs) of view counted to quantify the number of migrated cells. Data shown are mean $\pm S$.E. of independent experiments $(n=7) .{ }^{*} P<0.05$ ANOVA with Tukey's post-hoc test. (b) Chemokinesis control where the chemotactic gradient was disrupted via inclusion of microparticles in both upper and lower chambers. Data shown are mean \pm S.E. of independent experiments $(n=4)$. ${ }^{*} P<0.05$ ANOVA with Tukey's post-hoc test

and here we consider the role of ICAM-3 within the whole clearance process.

Here we report a novel mAb, MA4, that reacts with domains 1 and 2 of ICAM-3 and blocks interaction of apoptotic leukocytes with macrophages. In addition, we have developed cell lines deficient for ICAM-3 expression. The capacity of these ICAM-3-deficient cells, when apoptotic, for clearance by macrophages is reduced compared with their ICAM-3-replete counterparts and provides another powerful tool for ICAM-3 analysis. These ICAM-3-deficient cells complement previously described overexpression studies in HEK-293 cells where exogenous ICAM-3 expression promotes apoptotic cell clearance. ${ }^{17}$ Using these tools we demonstrate that ICAM-3 promotes apoptotic cell clearance by, at least minimally, tethering cell corpses to phagocytes. However, a role for ICAM-3 in mediating phagocytosis cannot be ruled out.

Apoptotic cell clearance has been suggested to occur through the development of a complex phagocytic synapse between phagocytes and dying cells, ${ }^{1}$ which is conceptually similar to the ICAM-3-containing immune synapse between $\mathrm{T}$ cells and antigen-presenting cells. This synapse also shares similarities with the sequential development of cellcell interactions required for efficient leukocyte-endothelial cell interaction before extravasation; here, weak interactions 
lead, via signalling events, to more stable interactions utilising $\beta 2$-integrins that arrest leukocytes on vascular walls through interaction with adhesion molecules including IgSF members. Such comparisons may be of significant relevance when describing the nature of the apoptotic cell-phagocyte interaction process, especially with a focus on ICAM-3, a further IgSF member that is leukocyte restricted.

The demonstration that ICAM-3 functions, on the apoptotic cell, as an adhesion molecule promoting the tethering of apoptotic leukocytes in a manner that is at least partially nonredundant is novel and expands our knowledge as to the involvement of the IgSF in this clearance process. Furthermore, it may provide useful evidence for the identification of the specific receptor that may be involved in the recognition of apoptotic cell-associated ICAM-3, a receptor that to date has proved elusive.

An intriguing aspect to ICAM-3 is its reduction in expression during apoptosis despite its novel tethering role in clearance. ${ }^{17}$ We show that this reduction in ICAM-3 is rapid and extensive. Apoptosis-associated disruption/masking of epitopes through molecular associations (e.g., ICAM-3 associations with itself or other molecules) or molecular changes (e.g., alterations in glycosylation) could reveal apparent reductions in ICAM-3 via mAb-based detection. Furthermore, shedding of ICAM-3 extracellular domains as a soluble cleavage product or release in apoptotic bodies may explain such observations. Here we used ICAM-3-GFP to monitor ICAM-3 directly and demonstrate in transfected HeLa cells that ICAM- 3 is present within surface blebs during apoptosis. This experimental system with exogenous expression of a tagged ICAM-3 within HeLa cells demonstrated that an intracellular GFP tag did not disrupt the ability of ICAM-3 to function as a tethering ligand on apoptotic cells. Notably, although overexpression may increase the removal of apoptotic non-leukocytes (HEK cells), ${ }^{17}$ it sometimes failed to increase tethering to phagocytes but simply introduced a new tethering mechanism, now inhibitable with anti-ICAM-3 mAb MA4.

The outcome of cell-cell interactions is dictated by the net effect of multiple systems acting between cells, including molecules that promote clearance and molecules that inhibit clearance. Notably, ICAM-3 is present in both viable and apoptotic leukocytes, although viable leukocytes are not tethered or engulfed by phagocytes. ${ }^{17}$ The novel role in corpse tethering and clearance for apoptotic cell-associated ICAM-3 is likely associated with a loss of molecules such as CD31 and CD47 that exert inhibitory effects to prevent viable cell clearance ${ }^{18,21}$ and may also include qualitative changes in ICAM-3 as cells proceed through apoptosis. Future work may assess the potential for native ICAM-3 on viable cells to act in an inhibitory manner similar to its close relative CD31.

Our evidence that ICAM-3 levels on leukocytes were reduced in line with reductions in cell volume and the observations of ICAM-3 within apoptotic ICAM-3-transfected HeLa cell blebs led us to identify ICAM-3 within microparticles released from apoptotic leukocytes. Such membrane loss has been demonstrated previously and is more than a simple phenomenon associated with poor membrane and cytoskeletal regulation during death. It is an important functional event for dying cells involved in phagocyte recruitment to undertake the final clearance step of the apoptosis programme. ${ }^{24}$
Microparticles from apoptotic BL cells, as used in our studies, have been reported to be important in monocyte recruitment to B-cell apoptosis in a manner that is CX3CL1 (fractalkine) dependent. ${ }^{25}$ CX3CL1 is a novel class of chemokine with adhesion properties, suggesting that it may play a dual role in monocyte recruitment, acting as both chemokine and adhesion molecule. Our data suggest that ICAM-3, a classical adhesion molecule, may also function within this process as ICAM-3-containing microparticles are significantly more potent in recruiting macrophages than their ICAM-3-deficient counterparts. Given our described role for ICAM-3 in tethering apoptotic cells to phagocytes, the established role for ICAM-3 in stabilising T cell-APC interactions and the involvement of other IgSF members (ICAM-1/2) in processes that require chemokine signalling (e.g., leukocyte extravasation), we propose that ICAM-3 may function as an adhesion molecule to complement the role of CX3CL1 or other microparticleassociated chemoattractants in recruiting phagocytes to sites of B-cell death. Indeed, it is likely that a range of chemoattractants are housed on and within complex microparticles and these have been suggested to act as long-distance mediators of attraction. ${ }^{32,33}$

Interestingly, evidence exists for preferential loss of ICAM-3 (54\% reduction) over CD31 (18\% reduction) in apoptotic neutrophils (which lose relatively little membrane during apoptosis relative to lymphocytes), suggesting ICAM-3 may be preferentially released in microparticles. ${ }^{34}$ Our work suggests this may be an active mechanism to recruit macrophages. Further work is also needed to assess the immunomodulatory effect of ICAM-3 and microparticles released from apoptotic cells. Our current work indicates that microparticles can exert anti-inflammatory effects on macrophages irrespective of their ICAM-3 expression, although preliminary data suggest that ICAM-3-replete microparticles may more rapidly exert their effect, perhaps through more efficient interaction of microparticles with phagocytes.

Our work raises the possibility that ICAM-3 may be targeted to modulate macrophage/monocyte recruitment to sites where leukocyte cell death may be occurring. The atherosclerotic plaque, a nonresolving inflammatory site, comprises a complex scenario of monocyte recruitment, overwhelmed capacity for phagocytic removal of lipid-laden apoptotic ('foam') cells coupled with further cell death and further recruitment in a progressive and deleterious pathological manner. ${ }^{35}$ Although one might expect ICAM-3 to be involved in monocyte recruitment to apoptotic foam cells, with monocytes expressing ICAM-3, it is perhaps even more compelling given the observations that foam cells express CX3CL $1,{ }^{36}$ and monocyte subsets in mice that accumulate in plaques express and utilise CX3CR 1 in their recruitment. ${ }^{37}$ Furthermore, it has recently been hypothesised that apoptotic cell-derived chemoattractants may recruit monocytes to tumours where as tumour-associated macrophages they play an important role in driving tumourigenesis. ${ }^{33}$ This model may be of great importance in the case of non-Hodgkin's lymphoma (e.g., Burkitt's lymphoma from where our Mutu BL cells derive) where high levels of apoptosis and large numbers of macrophages coexist. ${ }^{33}$

In conclusion, we demonstrate that ICAM-3 functions at least minimally as a ligand to tether apoptotic cells to 
phagocytes in a manner that is, at least partially, nonredundant. Associated with this function is a loss of ICAM-3 during apoptotic cell blebbing, and released ICAM-3-containing microparticles potently recruit phagocytes via chemotaxis to apoptotic leukocytes. Our data are consistent with ICAM-3 complementing the role of other chemoattractants (e.g., CX3CL1) and may function as an important adhesion molecule within this recruitment pathway. Further work is now needed to assess the importance of ICAM-3 in recruiting phagocytes to sites of leukocyte cell death (e.g., atherosclerotic plaques and leukocyte-derived tumours) and to characterise the exact molecular mechanisms by which it appears to exert its effect.

\section{Materials and Methods}

Cell isolation, cell lines and culture. Anti-ICAM-3-expressing hybridoma cells were produced by fusing spleen cells from ICAM-3/HEK transfectant ${ }^{17}$ immunised Balb/c mice with Ag8.653 cells. All work was undertaken in accordance with regulatory guidance. Briefly, splenocytes from an immunised mouse were fused (5:1 ratio) with myeloma cells in the presence of 50\% PEG1500 (Roche, Applied Science, West Sussex, UK) under serum-free conditions. Hybridomas were cloned using limiting dilution in RPMl-1640 medium containing $2 \mathrm{mM} \mathrm{L-glutamine}$ supplemented with $10 \% \mathrm{v} / \mathrm{v}$ fetal calf serum (FCS; PAA, Yeovil, UK), $100 \mathrm{IU} / \mathrm{ml}$ penicillin and $100 \mu \mathrm{g} / \mathrm{ml}$ streptomycin) and HAT selection supplement (Sigma, Poole, UK). The anti-ICAM-3 reactivity of culture supernatants against immobilised ICAM-3-FC and CD14-Fc was assessed using ELISA.

Mutu I BL cells, ${ }^{38}$ Jurkat (human T) and THP-1 (human myelomonocytic) were cultured in RPMl-1640 medium containing $2 \mathrm{mM}$ L-glutamine supplemented with $10 \%$ FCS (PAA) and $100 \mathrm{lU} / \mathrm{ml}$ penicillin and $100 \mu \mathrm{g} / \mathrm{ml}$ streptomycin). RAW 264.7 cells (murine macrophage ${ }^{39}$ ), J774 cells (murine macrophage ${ }^{40}$ ), HeLa 229 (human epithelial) and HEK-293 cells (human epithelial) cells were cultured similarly but in DMEM (PAA) supplemented with FCS, glutamine and antibiotics as above.

ICAM-3-deficient Mutu I cells were generated by sequential fluorescenceactivated cell sorting of a WT Mutu I population. Briefly, cells were stained aseptically with excess anti-ICAM-3 mAb (CAL3.10; R\&D Systems, Abingdon, UK) followed by goat anti-mouse FITC (Sigma). At least 10000 viable cells from within the lowest $5 \%$ fluorescence region were collected using fluorescence-activated cell sorting. Cells were recovered in culture for 1 to 2 weeks before repeat sorting. Three rounds of flow cytometric sorting were used to produce stable, non-ICAM-3expressing cell populations. ICAM-3 expression was assessed regularly using indirect immunofluorescence to ensure no spontaneous recovery of ICAM-3 on ICAM-3-deficient sorted populations.

Monoclonal antibody-based assays. Monoclonal antibodies were produced as outlined above and clones of interest were further cultured and tissue culture supernatant harvested from static cultures when cells were $+70 \%$ apoptotic. Such tissue culture supernatants were routinely used for subsequent analyses.

For ELISA, Nunclon Maxisorp plates (Thermo-Fisher Scientific, Loughborough, UK) were coated overnight with sheep anti-human Fc (The Binding Site, Birmingham, UK) at $5 \mu \mathrm{g} / \mathrm{ml}$ in carbonate buffer (pH 9.6; Sigma). Following washing in PBS(T) (phosphate-buffered saline $+0.05 \% \mathrm{v} / \mathrm{v}$ Tween-20), Fc-tagged proteins were added at a concentration of $1 \mu \mathrm{g} / \mathrm{ml}$ for $1 \mathrm{~h}$ at $37^{\circ} \mathrm{C}$. The resultant, oriented $\mathrm{Fc}$ fusions were probed with $\mathrm{mAbs}$ and bound $\mathrm{mAb}$ detected using antimouse HRP (GE Healthcare, Buckinghamshire, UK) and colourimetric SigmaFAST OPD assay (Sigma).

For western blot analysis, cells were solubilised under reducing and denaturing conditions, separated using standard polyacrylamide gel electrophoresis and electroblotted to nitrocellulose $(0.45 \mu \mathrm{m}$, Schleicher \& Schuell, Dassel, Germany, supplied by Thermo-Fisher Scientific). Membranes were blocked in $5 \%$ non-fat milk powder in TBS-tween $(0.05 \% \mathrm{v} / \mathrm{v} ; \mathrm{pH} 7.2)$, probed with mAbs and detected using anti-mouse HRP and ECL detection kits (GE Healthcare).

For indirect immunofluorescence, cells were incubated with excess mAb on ice for $30 \mathrm{~min}$, washed twice in PBS(A)/BSA $(0.5 \% \mathrm{w} / \mathrm{v})$ and incubated with goat anti-mouse FITC ( $1 \mu$ l per 200000 cells in $100 \mu$ volume; Sigma). Stained cells were analysed either directly or following fixation in $1 \% \mathrm{w} / \mathrm{v}$ formaldehyde in PBS(A) using a Beckman-Coulter Quanta SC flow cytometer (Beckman Coulter, High Wycombe, UK). Downstream flow cytometric analyses and presentations were undertaken using VenturiOne software from Applied Cytometry Systems (Sheffield, UK) or FlowJo (Treestar Inc., Ashland, OR, USA).

Production of recombinant human CD14 and ICAM-3. Recombinant human CD14 or human ICAM-3 were produced as soluble Fc fusion proteins (fused to $\mathrm{CH} 2 / \mathrm{CH} 3$ domains of human IgG1). ${ }^{17}$ DNA for the recombinant proteins was transfected to 293 cells using standard calcium phosphate-mediated transfections. Fc fusion proteins were purified from culture supernatants using HiTrap protein $G$ columns (GE Healthcare).

ICAM-3-GFP (ICAM-3 with a carboxy terminal EmGFP tag) was constructed using the Vivid Colours pcDNA6.2/C-EmGFP-GW/TOPO mammalian expression vector (Life Technologies, Paisley, UK) as per the manufacturer's instructions, with forward primer: 5'-ATTTAAGCTTATGGCCACCATGGTACCA-3' and reverse primer: $5^{\prime}$-TTTTGCGGCCGCTATCATTACTTGTACAGCTCG-3'. The sequence of ICAM3-GFP was confirmed by sequencing (Functional Genomics, Birmingham, UK). In order to express ICAM-3-GFP in HeLa cells, DNA was transfected using the TransITLT1 transfection reagent (Mirus Bio Corporation, Madison, WI, USA; supplied by Geneflow, Fradley, Staffordshire, UK) as per the manufacturer's instructions.

Apoptosis induction and quantification. Mutu BL or Jurkat cells were subjected to a measured dose of UV-B irradiation, using a Chromata-vue C71 light box and UVX radiometer (UV-P Inc., Upland, CA, USA) to induce apoptosis. BL cells received $100 \mathrm{~mJ} / \mathrm{cm}^{2}$ whereas Jurkats received $150 \mathrm{~mJ} / \mathrm{cm}^{2}$. For analysis of apoptotic nuclear morphology, cells were fixed in $1 \%$ formaldehyde, stained with 4,6-diamidino-2-phenylindole (DAPI, Sigma, $250 \mathrm{ng} / \mathrm{ml}$ ) and observed using inverted epifluorescence microscopy. For quantitative analyses, percentages of apoptotic cells per $\geqslant 200$ counted per sample were enumerated. HeLa cells were induced with anisomycin (Sigma; $5 \mu \mathrm{g} / \mathrm{ml} \mathrm{from} \mathrm{a} \mathrm{stock} \mathrm{of} 5 \mathrm{mg} / \mathrm{ml}$ in DMSO). HeLa cell apoptosis was assessed by analysis of nuclear morphology following staining with DAPI and epifluorescence microscopy. Photomicrography was undertaken using a fully motorised Zeiss Axiovert $200 \mathrm{M}$ fluorescence microscope (Carl Zeiss Ltd, Welwyn Garden City, Fradley, Staffordshire, UK) and Hamamatsu Orca camera driven by Volocity (Perkin-Elmer, Cambridge, UK).

Annexin V labelling and flow cytometry. Cells were stained with annexin V-FITC (eBioscience Ltd., Hatfield, UK). Briefly, cells were washed and resuspended in binding buffer (10 mM HEPES pH 7.4, $150 \mathrm{mM} \mathrm{NaCl}, 2.5 \mathrm{mM}$ $\mathrm{CaCl}_{2}$ ) containing annexin V-FITC ( $1 \mu \mathrm{l}$ per 200000 cells) for $15 \mathrm{~min}$ on ice. Cells were washed once in binding buffer and diluted to $1 \mathrm{ml}$ with binding buffer and PI added to a final concentration of $20 \mu \mathrm{g} / \mathrm{ml}$. Samples were analysed immediately on a Quanta SC flow cytometer (Beckman Coulter).

Assays of phagocyte interaction with apoptotic cells. Assay of interaction (binding and phagocytosis) of phagocytes (RAW, J774 or THP-1, primary human monocyte-derived macrophages) with apoptotic cells was carried out either on multiwell glass slides ${ }^{26}$ or in 24-well plates as described. ${ }^{29}$ Briefly, for the slidebased assay, apoptotic cells and phagocytes (100:1 ratio) were co-cultured for $1 \mathrm{~h}$ at $37^{\circ} \mathrm{C}$ in RPMl containing $0.2 \%$ (w/v) bovine serum albumin (Sigma). Unbound cells were removed by extensive washing and slides fixed in methanol, stained with Jenner/Giemsa (Raymond Lamb, Thermo-Fisher Scientific) and mounted in DePeX (VWR, Lutterworth, UK) before examination by light microscopy. Assays were performed similarly in 24-well plates but at the end of the procedure, cells were fixed in $1 \%$ formaldehyde and stained with DiffQuick II (Medion Diagnostics $\mathrm{GmbH}$, Dudingen, Switzerland). In all cases, at least 200 macrophages were assessed in each of at least triplicate wells. As appropriate, antibodies were included in a 1:10 dilution of tissue culture supernatant. For assay of binding/tethering, co-cultures were carried out at room temperature $\left(20^{\circ} \mathrm{C}\right)$. Data are presented as percent macrophage binding/tethering or interacting with apoptotic cells.

Where THP-1 cells were used as the phagocyte source, cells were cultured for $72 \mathrm{~h}$ in the presence of dihydroxyvitamin D3 (Enzo Life Sciences, Exeter, UK, $100 \mathrm{nM}$ ) and/or phorbol-12-myristate-13-acetate (PMA, $250 \mathrm{nM}$; Sigma) in four-well glass slides (Hendley (Essex) Ltd, Loughton, UK). Where primary human monocytederived macrophages were used, monocytes were isolated from peripheral blood of healthy volunteers and cultured for 7 days in the presence of $10 \%$ autologous serum in Iscove's modified Dulbecco's medium. ${ }^{26}$ 
Isolation of microparticles and chemotaxis assays. To prepare microparticles, viable cells were resuspended at $2 \times 10^{6}$ viable cells $/ \mathrm{ml}$ in serum-free RPMI supplemented with BSA $(0.1 \%$ w/v), $2 \mathrm{mM} \mathrm{L-glutamine} \mathrm{and}$ $100 \mathrm{IU} / \mathrm{ml}$ penicillin and $100 \mu \mathrm{g} / \mathrm{ml}$ streptomycin. Following induction to apoptosis and $16 \mathrm{~h}$ of incubation, supernatants were harvested. To remove cell bodies, supernatants were subject to low-speed centrifugation $(7 \mathrm{~min} ; 350 \times \mathrm{g})$ and lack of cells confirmed with microscopy. Supernatants were now used in chemotaxis assays either neat or diluted with RPMI/BSA $(0.1 \% \mathrm{w} / \mathrm{v})$. To purify microparticles for Western blot analysis, supernatants were further centrifuged $(30 \mathrm{~min} ; 14000 \times \mathrm{g})$.

For the chemotaxis assay, a 48-well chemotaxis chamber (Neuroprobe Inc. Gaithersburg, Madison, WI, USA) was used in line with the manufacturer's instructions. Briefly, $25 \mu \mathrm{l}$ of putative chemoattractant was loaded in the lower chamber, covered with PVP-free polycarbonate membrane $(5 \mu \mathrm{m}$ pore size, $4 \times 10^{3}$ pores $\left./ \mathrm{mm}^{2}\right)$ and $50 \mu \mathrm{l}\left(1 \times 10^{5}\right.$ cells $)$ of phagocytes added to the upper chamber. Cell migration was allowed to proceed at $37^{\circ} \mathrm{C}$ for $4 \mathrm{~h}$ before washing and staining of the membrane. Migrated cells remained adherent to the membrane (bottom side) and were counted using light microscopy. In order to expose phagocytes to microparticles in the absence of a concentration gradient, microparticles were included in both chambers.

\section{Conflict of Interest}

The authors declare no conflict of interest.

Acknowledgements. We are grateful to Professor Yvonne Perrie for support in particle sizing; Steve Wells for expert technical assistance; Professor Christopher D Gregory (Edinburgh University) for provision of BL cell lines; and Dr. David L Simmons (Cellzome formerly of University of Oxford) for ICAM-3-Fc expression constructs. Thanks are also to the BBSRC for funding to support EET. AD was in receipt of a BBSRC new Investigator Award (BB/E002080/1).

1. Savill J, Dransfield I, Gregory C, Haslett C. A blast from the past: clearance of apoptotic cells regulates immune responses. Nat Rev Immunol 2002; 2: 965-975.

2. Gregory $C D$, Devitt $A$. The macrophage and the apoptotic cell: an innate immune interaction viewed simplistically? Immunology 2004; 113: 1-14.

3. Henson PM, Hume DA. Apoptotic cell removal in development and tissue homeostasis Trends Immunol 2006; 27: 244-250.

4. Erwig LP, Henson PM. Clearance of apoptotic cells by phagocytes. Cell Death Differ 2008; 15: 243-250.

5. Lauber K, Blumenthal SG, Waibel M, Wesselborg S. Clearance of apoptotic cells: getting rid of the corpses. Mol Cell 2004; 14: 277-287.

6. Peter C, Waibel M, Radu CG, Yang LV, Witte ON, Schulze-Osthoff K et al. Migration to apoptotic "find-me" signals is mediated via the phagocyte receptor G2A. J Biol Chem 2008 283: 5296-5305.

7. Elliott MR, Chekeni FB, Trampont PC, Lazarowski ER, Kadl A, Walk SF et al. Nucleotides released by apoptotic cells act as a find-me signal to promote phagocytic clearance. Nature 2009; 461: 282-286.

8. Gregory C. Cell biology: sent by the scent of death. Nature 2009; 461: 181-182.

9. Fadok VA, Voelker DR, Campbell PA, Cohen JJ, Bratton DL, Henson PM. Exposure of phosphatidylserine on the surface of apoptotic lymphocytes triggers specific recognition and removal by macrophages. J Immunol 1992; 148: 2207-2216.

10. Verhoven B, Schlegel RA, Williamson P. Mechanisms of phosphatidylserine exposure a phagocyte recognition signal, on apoptotic T lymphocytes. J Exp Med 1995; 182 1597-1601

11. Fadok VA, de Cathelineau A, Daleke DL, Henson PM, Bratton DL. Loss of phospholipid asymmetry and surface exposure of phosphatidylserine is required for phagocytosis of apoptotic cells by macrophages and fibroblasts. J Biol Chem 2001 276: 1071-1077.

12. Dini L, Autuori F, Lentini A, Oliverio S, Piacentini M. The clearance of apoptotic cells in the liver is mediated by the asialoglycoprotein receptor. FEBS Lett 1992; 296: 174-178.

13. Dini L. Recognizing death: liver phagocytosis of apoptotic cells. Eur J Histochem 2000; 44 217-227.

14. Duvall $E$, Wyllie AH, Morris RG. Macrophage recognition of cells undergoing programmed cell death (apoptosis). Immunology 1985; 56: 351-358.

15. Kagan VE, Gleiss B, Tyurina YY, Tyurin VA, Elenstrom-Magnusson C, Liu SX et al. A role for oxidative stress in apoptosis: oxidation and externalization of phosphati- dylserine is required for macrophage clearance of cells undergoing Fas-mediated apoptosis. J Immunol 2002; 169: 487-499.

16. Arur S, Uche UE, Rezaul K, Fong M, Scranton V, Cowan AE et al. Annexin I is an endogenous ligand that mediates apoptotic cell engulfment. Dev Cell 2003; 4: 587-598.

17. Moffatt OD, Devitt A, Bell ED, Simmons DL, Gregory CD. Macrophage recognition of ICAM-3 on apoptotic leukocytes. J Immunol 1999; 162: 6800-6810.

18. Brown S, Heinisch I, Ross E, Shaw K, Buckley CD, Savill J. Apoptosis disables CD31mediated cell detachment from phagocytes promoting binding and engulfment. Nature 2002; 418: 200-203.

19. Vernon-Wilson EF, Aurade F, Brown SB. CD31 promotes beta1 integrin-dependent engulfment of apoptotic Jurkat $T$ lymphocytes opsonized for phagocytosis by fibronectin. J Leukoc Biol 2006; 79: 1260-1267.

20. Vernon-Wilson EF, Aurade F, Tian L, Rowe IC, Shipston MJ, Savill J et al. CD31 delays phagocyte membrane repolarization to promote efficient binding of apoptotic cells. $J$ Leukoc Biol 2007; 82: 1278-1288.

21. Gardai SJ, McPhillips KA, Frasch SC, Janssen WJ, Starefeldt A, Murphy-Ullrich JE et al. Cell-surface calreticulin initiates clearance of viable or apoptotic cells through transactivation of LRP on the phagocyte. Cell 2005; 123: 321-334.

22. Gardai SJ, Bratton DL, Ogden CA, Henson PM. Recognition ligands on apoptotic cells: a perspective. J Leukoc Biol 2006; 79: 896-903.

23. Nilsson A, Oldenborg PA. CD47 promotes both phosphatidylserine-independent and phosphatidylserine-dependent phagocytosis of apoptotic murine thymocytes by nonactivated macrophages. Biochem Biophys Res Commun 2009; 387: 58-63.

24. Segundo C, Medina F, Rodriguez C, Martinez-Palencia R, Leyva-Cobian F, Brieva JA. Surface molecule loss and bleb formation by human germinal center $B$ cells undergoing apoptosis: role of apoptotic blebs in monocyte chemotaxis. Blood 1999; 94: 1012-1020.

25. Truman LA, Ford CA, Pasikowska M, Pound JD, Wilkinson SJ, Dumitriu IE et al. CX3CL1/ fractalkine is released from apoptotic lymphocytes to stimulate macrophage chemotaxis. Blood 2008; 112: 5026-5036.

26. Devitt A, Moffatt OD, Raykundalia C, Capra JD, Simmons DL, Gregory CD. Human CD14 mediates recognition and phagocytosis of apoptotic cells. Nature 1998; 392: 505-509.

27. Devitt A, Pierce S, Oldreive C, Shingler WH, Gregory CD. CD14-dependent clearance of apoptotic cells by human macrophages: the role of phosphatidylserine. Cell Death Differ 2003; 10: 371-382.

28. Griffiths HR, Willetts RS, Grant MM, Mistry N, Lunec J, Bevan RJ. In vivo vitamin C supplementation increases phosphoinositol transfer protein expression in peripheral blood mononuclear cells from healthy individuals. Br J Nutr 2009; 101: 1432-1439.

29. Devitt A, Parker KG, Ogden CA, Oldreive C, Clay MF, Melville LA et al. Persistence of apoptotic cells without autoimmune disease or inflammation in CD14-/- mice. J Cell Biol 2004; 167: 1161-1170.

30. Dive C, Gregory CD, Phipps DJ, Evans DL, Milner AE, Wyllie AH. Analysis and discrimination of necrosis and apoptosis (programmed cell death) by multiparameter flow cytometry. Biochim Biophys Acta 1992; 1133: 275-285.

31. Lane JD, Allan VJ, Woodman PG. Active relocation of chromatin and endoplasmic reticulum into blebs in late apoptotic cells. J Cell Sci 2005; 118 (Pt 17): 4059-4071.

32. Mause SF, Weber C. Microparticles: protagonists of a novel communication network for intercellular information exchange. Circ Res 2010; 107: 1047-1057.

33. Gregory CD, Pound JD. Microenvironmental influences of apoptosis in vivo and in vitro. Apoptosis 2010; 15: 1029-1049.

34. Hart SP, Ross JA, Ross K, Haslett C, Dransfield I. Molecular characterization of the surface of apoptotic neutrophils: implications for functional downregulation and recognition by phagocytes. Cell Death Differ 2000; 7: 493-503.

35. Tabas I. Macrophage death and defective inflammation resolution in atherosclerosis. Nat Rev Immunol 2010; 10: 36-46.

36. Barlic J, Murphy PM. Chemokine regulation of atherosclerosis. J Leukoc Biol 2007; 82: 226-236.

37. Tacke F, Alvarez D, Kaplan TJ, Jakubzick C, Spanbroek R, Llodra J et al. Monocyte subsets differentially employ CCR2, CCR5, and CX3CR1 to accumulate within atherosclerotic plaques. J Clin Invest 2007; 117: 185-194.

38. Gregory $C D$, Rowe M, Rickinson $A B$. Different Epstein-Barr virus-B cell interactions in phenotypically distinct clones of a Burkitt's lymphoma cell line. J Gen Virol 1990; 71 (Pt 7): 1481-1495

39. Raschke WC, Baird S, Ralph P, Nakoinz I. Functional macrophage cell lines transformed by Abelson leukemia virus. Cell 1978; 15: 261-267.

40. Ralph P, Nakoinz I. Phagocytosis and cytolysis by a macrophage tumour and its cloned cell line. Nature 1975; 257: 393-394.

This work is licensed under the Creative Commons Attribution-NonCommercial-No Derivative Works 3.0

Unported License. To view a copy of this license, visit http:/l creativecommons.org/licenses/by-nc-nd/3.0

Supplementary Information accompanies the paper on Cell Death and Differentiation website (http://www.nature.com/cdd) 